\title{
Utilizing engineered nucleation features to increase productivity in protein crystallization trials
}

Tiffany L. Kinnibrugh , Kyle A. Nordquist, Kevin M. Schaab, Youngchang Kim, Gyorgy Babnigg, and Andrew H. Bond*

DeNovX, 3440 S. Dearborn St., Lab 204 S, Chicago, IL 60616

Structural Biology Center, Biosciences Division, Bldg. 446, Argonne National Laboratory, 9700

S. Cass Ave., Argonne, IL 60439

Improving the outcomes of protein crystallization trials has become increasingly important in minimizing crystallization as a key bottleneck to protein structure determination. Crystallization success rates are often $<20 \%$ even in the most successful laboratories, which leaves significant room for improvements in crystallization outcomes (e.g., crystal hit \%, quantity, crystallization onset time, etc.). Enhancements to crystallization success rates have been achieved by modifying the surface energy of the crystallization vessel to facilitate interactions between the surface and the solutes to be crystallized. By systematically varying the energy of the crystallization surfaces, DeNovX has demonstrated an $11-87 \%$ reduction in crystallization onset times with an average $2.5 \pm 0.4$-fold increase in crystal quantity vs. surface controls for benchmark proteins (e.g., lysozyme, bovine pancreatic trypsin, etc.) and proteins from the pipeline at the Midwest Center for Structural Genomics at Argonne National Laboratory. Replicate studies show that an average of $70 \pm 18 \%$ of crystals form on, or in contact with, the engineered nucleation features, which suggests that these features play an important role in crystal nucleation. Importantly, the quality metrics for structure solution of proteins crystallized using engineered nucleation features are similar to the analogous PDB entries. Rigorously controlled, replicate studies have been carried out across a variety of crystallization form factors (e.g., microscope slides, HTS screening plates, cover slips, vials, capillaries, etc.) with different techniques (e.g., sitting drop vapor diffusion, hanging drop vapor diffusion, batch studies) and have demonstrated that surface energy modifications can be an effective way to improve crystallization outcomes while requiring minimal changes to existing workflows. These crystallization improvements can reduce experimental time and costs, and may improve structure determination rates for high impact protein targets relevant to disease progression and drug development.

Tiffany Kinnibrugh tkinnibrugh@denovx.com

Kyle Nordquist knordquist@denovx.com

Kevin Schaab kschaab@denovx.com

Youngchang Kim ykim@anl.gov

Gyorgy Babnigg gbabnigg@anl.gov

Andrew Bond abond@denovx.com 